MADPH-97-1009

July 1997

\title{
THE MUON ELECTRIC DIPOLE MOMENT
}

\author{
Vernon Barger, Chung Kao \\ Department of Physics, University of Wisconsin, \\ Madison, WI 53706, USA \\ Ashok Das \\ Department of Physics and Astronomy, University of Rochester, \\ Rochester, NY 14627, USA
}

\begin{abstract}
The electric dipole moment of the muon $\left(d_{\mu}\right)$ is evaluated in a two Higgs doublet model with a softly broken discrete symmetry. For $\tan \beta \equiv\left|v_{2}\right| /\left|v_{1}\right| \sim 1$, contributions from two loop diagrams involving the $t$ quark and the $W$ boson dominate; while for $\tan \beta \gtrsim 10$, contributions from two loop diagrams involving the $b$ quark and the $\tau$ lepton are dominant. For $8 \gtrsim \tan \beta \gtrsim 4$, significant cancellation occurs among the contributions from two loop diagrams and the one loop contribution dominates for $\tan \beta \sim 7$. For $\tan \beta \gtrsim 15$, the calculated $d_{\mu}$ can be close to the reach of a recently proposed experiment at the Brookhaven National Laboratory.
\end{abstract}

\section{Introduction}

For a particle with spin $\vec{s}$, and an electric dipole moment (EDM) $\vec{d} \propto \vec{s}$, the interaction Hamiltonian has the following form:

$$
H_{I}=-\vec{d} \cdot \vec{E},
$$

where $\vec{E}$ is an electric field. This Hamiltonian violates both parity $(\mathrm{P})$ invariance and time-reversal $(\mathrm{T})$ invariance. The invariance of CPT implies that the combined transformation of charge conjugation $(\mathrm{C})$ and parity $(\mathrm{P})$ is not an exact symmetry. 1 . The EDM of a fermion $\left(d_{f}\right)$ can be described with an effective Lagrangian

$$
\mathcal{L}_{\text {eff }}=-\frac{i}{2} d_{f} \bar{\psi} \sigma_{\mu \nu} \gamma_{5} \psi F^{\mu \nu}
$$

In the non-relativistic limit, the Lagrangian becomes $\mathcal{L}_{\text {eff }}=-H_{I}=d_{f} \vec{s} \cdot \vec{E}$.

In the Standard Model (SM) of electroweak interactions, CP violation is generated by the Kobayashi-Maskawa (KM) phase. The electron EDM $\mathrm{B}$ is about $8 \times 10^{-41} \mathrm{e} \cdot \mathrm{cm}$ and the expected muon EDM is about $2 \times 10^{-38} \mathrm{e} \cdot \mathrm{cm}$. Several experiments have been carried out to search for an electron EDM $\left(d_{e}\right)$

* Talk presented by C. Kao at the 2nd International Conference on B Physics and CP Violation, Honolulu, Hawaii, USA, March 1997. 
and a muon $\operatorname{EDM}\left(d_{\mu}\right)$. At $95 \%$ C.L., the experimental upper limits on $d_{e}$ and $d_{\mu}$ are (i) $\left|d_{e}\right|<6.2 \times 10^{-27} \mathrm{e} \cdot \mathrm{cm}$, and (ii) $\left|d_{\mu}\right|<1,1 \times 10^{-18} \mathrm{e} \cdot \mathrm{cm}$.

Recently, a dedicated experiment has been proposed 6 to measure the muon electric dipole moment at the Brookhaven National Laboratory. This experiment will be able to improve the measurement of the muon EDM by at least 4 orders of magnitude.

\section{CP Violation from Higgs Exchange}

In multi-Higgs doublet models, a discrete symmetry is usually required for flavor symmetry to be conserved. In two Higgs doublet models, this discrete symmetry is often chosen to be $\phi_{1} \rightarrow-\phi_{1}$ and $\phi_{2} \rightarrow+\phi_{2}$. If this discrete symmetry is only softly broken 8 , not only can Higgs boson exchange generate $\mathrm{CP}$ violation but also flavor changing neutral Higgs interactions can be kept at an acceptable level.

A two Higgs doublet model has doublets $\phi_{1}$ and $\phi_{2}$. After spontaneous symmetry breaking, there remain five physical 'Higgs bosons': a pair of singly charged Higgs bosons $\left(H^{ \pm}\right)$, two neutral CP-even scalars $\left(H_{1}\right.$ and $\left.H_{2}\right)$, and a neutral CP-odd pseudoscalar $(A)$. There are two sources of $\mathrm{CP}$ violation in the Higgs potential: (i) the mixing of the $A$ with the $H_{1}$ and the $H_{2}$, and (ii) the $\mathrm{CP}$ violating interaction of $A H^{+} H^{-}$.

Adopting Weinberg's parameterization 1 we can write the following neutral Higgs exchange propagators as

$$
\begin{aligned}
<H_{1} A>_{q} & =\frac{1}{2} \sum_{n} \frac{\sin 2 \beta \operatorname{Im} Z_{0 n}}{q^{2}-m_{n}^{2}} \\
& =\frac{1}{2} \sum_{n}\left[-\frac{\cos ^{2} \beta \cot \beta \operatorname{Im} \tilde{Z}_{1 n}}{q^{2}-m_{n}^{2}}+\frac{\sin ^{2} \beta \tan \beta \operatorname{Im} \tilde{Z}_{2 n}}{q^{2}-m_{n}^{2}}\right] \\
<H_{2} A>_{q} & =\frac{1}{2} \sum_{n} \frac{\cos 2 \beta \operatorname{Im} Z_{0 n}-\operatorname{Im} \tilde{Z}_{0 n}}{q^{2}-m_{n}^{2}} \\
& =\frac{1}{2} \sum_{n} \frac{\cos ^{2} \beta \operatorname{Im} \tilde{Z}_{1 n}+\sin ^{2} \beta \operatorname{Im} \tilde{Z}_{2 n}}{q^{2}-m_{n}^{2}}
\end{aligned}
$$

where the summation is over all the mass eigenstates of neutral Higgs bosons. We will assume that the sums are dominated by the lightest neutral Higgs boson of mass $m_{0}$, and drop the sums and indices. There are relations among the CP violation parameters: $\operatorname{Im} Z_{0}+\operatorname{Im} \tilde{Z}_{0}=-\cot ^{2} \beta \operatorname{Im} \tilde{Z}_{1}$, and $\operatorname{Im} Z_{0}-\operatorname{Im} \tilde{Z}_{0}=$ $+\tan ^{2} \beta \operatorname{Im} \tilde{Z}_{2}$. Employing unitarity constraints, Weinberg has shown that $\left|\operatorname{Im} Z_{1}\right| \leq(1 / 2)|\tan \beta|\left(1+\tan ^{2} \beta\right)^{1 / 2}$ and $\left|\operatorname{Im} Z_{2}\right| \leq(1 / 2)|\cot \beta|\left(1+\cot ^{2} \beta\right)^{1 / 2}$. 


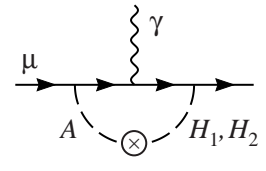

(a)

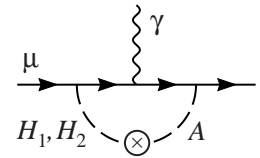

(b)

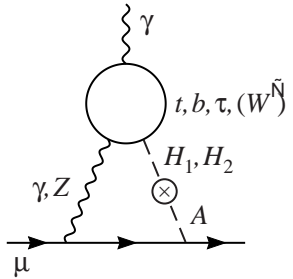

(c)

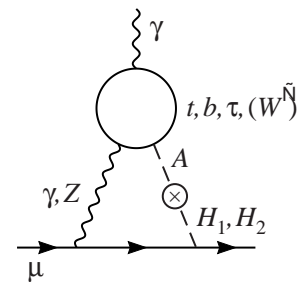

(d)

Figure 1: Feynman diagrams for leading contributions to the muon EDM.

\section{The Muon Electric Dipole Moment}

The Feynman diagrams contributing to the muon electric dipole moment are shown in Figure 1. There are many more diagrams involving the $W$ boson that are not shown in this figure. We consider only two-loop diagrams with an intermediate photon since the diagrams involving an intermediate $Z$ boson are highly suppressed by the vector part of the $Z e^{+} e^{-}$couplings.

In a two Higgs doublet model the electron EDM and the muon EDM have contributions from two-lopp diagrams containing the top quark $(t$-lopp) 10 , the gauge bosons ( $W$-loop) 11 , and the charged Higgs boson $(H$-loop $) 12$.

In the contribution from the charged Higgs boson, CP violation comes from both the neutral Higgs transition propagators and the trilinear vertex $A H^{+} H^{-}$.f For $\tan \beta$ larger than abput 10, there are dominant contributions from the $b$ quark and the $\tau$ lepton 14.

There are several interesting aspects: (i) The contributions from the $b$ and the $\tau$ loops are proportional to $\left(\operatorname{Im} Z_{0}+\operatorname{Im} \tilde{Z}_{0}\right)$. (ii) For the the $t$-loop, the coefficient of the $\operatorname{Im} \tilde{Z}_{0}$ is much smaller than that of the $\operatorname{Im} Z_{0}$. (iii) The $W$-loop does not contribute to the $\operatorname{Im} \tilde{Z}_{0}$ term.

The one-loop contribution to the muon $\operatorname{EDM}\left(d_{\mu}\right)$ is

$$
\begin{aligned}
d_{\mu}^{1-\text { loop }} & =\frac{e m \sqrt{2} G_{F} \tan ^{2} \beta}{(4 \pi)^{2}} I(\rho)\left(\operatorname{Im} Z_{0}+\operatorname{Im} \tilde{Z}_{0}\right) \\
I(\rho) & \equiv \rho \int_{0}^{1} d x \frac{x^{2}}{\rho x^{2}-x+1}
\end{aligned}
$$

where $\rho=m^{2} / m_{0}^{2}$. In the limit of $m_{0} \gg m$ (i.e. $\left.\rho \ll 1\right), I(\rho)$ approaches $-\rho[\ln (\rho)+3 / 2]$ and $d_{(e, \mu)}^{1-\text { loop }} \propto\left(m^{3} / m_{0}^{2}\right)\left[\ln \left(m^{2} / m_{0}^{2}\right)\right]$, for $m=m_{e}, m_{\mu}$.

$\dagger$ Contributions from the $H^{ \pm}$are not expected to be significant, since reqent CLEO measurements on $B(b \rightarrow s \gamma)$ constrain $m_{H \pm}$ to be at least $3 M_{W}$ in Model II. 13 


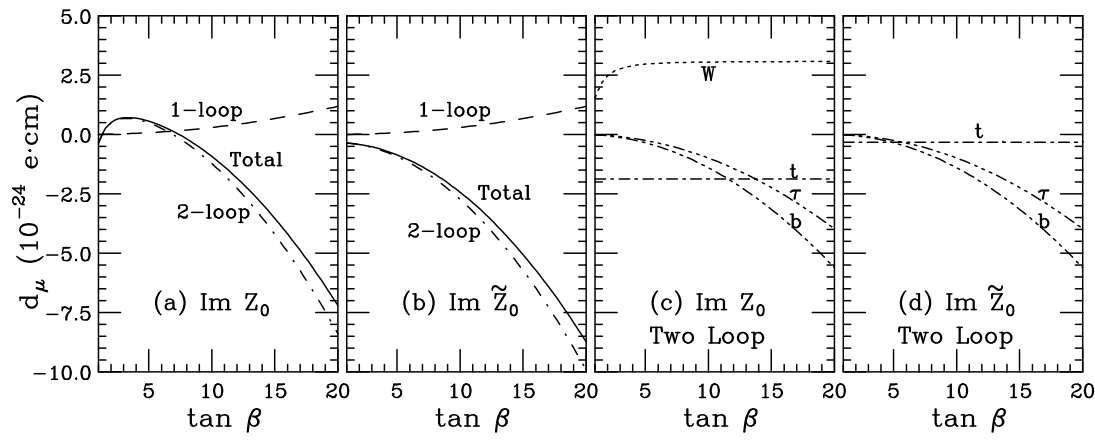

Figure 2: The muon EDM for $m_{0}=100 \mathrm{GeV}$ in units of $\operatorname{Im} Z_{0}$ and $\operatorname{Im} \tilde{Z}_{0}$.

Figure 2 shows the muon EDM generated from one-loop diagrams (dash), two-loop diagrams (dash-dot) and their total (solid) in units of (a) $\operatorname{Im} Z_{0}$ and (b) Im $\tilde{Z}_{0}$. Also shown are the contributions from two-loop diagrams involving the $W$ boson (dot), the $t$ quark (dash-dot), the $b$ quark (dash-dot-dot), and the $\tau$ lepton (dash-dot-dot-dot), in units of (c) $\operatorname{Im} Z_{0}$ and (d) $\operatorname{Im} \tilde{Z}_{0}$. This figure shows the effect of varying $\tan \beta$ on the muon EDM, for the case of $m_{0}=100$ $\mathrm{GeV}$. We note that: (i) for $\tan \beta$ close to one, contributions from two loop diagrams involving the top quark and the $W$ boson dominate; (ii) for $\tan \beta \gtrsim 10$ contributions from two loop diagrams involving the $b$ quark and the $\tau$ lepton are dominant; (iii) for $8 \gtrsim \tan \beta \gtrsim 4$, significant cancellation occurs among the contributions from two loop diagrams and one loop diagrams dominate for $\tan \sim 7$; (iv) for $\tan \beta \sim 1$ or $\tan \beta \gtrsim 10$, the muon and the electron EDMs satisfy a simple scaling relation $d_{\mu} \approx\left(m_{\mu} / m_{e}\right) d_{e} ;(\mathrm{v})$ for $8 \gtrsim \tan \beta \gtrsim 4,\left|d_{\mu}\right|$ can be two to three times $\left|\left(m_{\mu} / m_{e}\right) d_{e}\right|$.

The muon EDM can be expressed as

$$
\begin{aligned}
d_{\mu} & =d_{A} \operatorname{Im} Z_{0}+d_{B} \operatorname{Im} \tilde{Z}_{0} \\
& =\frac{d_{A}+d_{B}}{2}\left(\operatorname{Im} Z_{0}+\operatorname{Im} \tilde{Z}_{0}\right)+\frac{d_{A}-d_{B}}{2}\left(\operatorname{Im} Z_{0}-\operatorname{Im} \tilde{Z}_{0}\right),
\end{aligned}
$$

where $d_{A}$ and $d_{B}$ are the total coefficients of $\operatorname{Im} Z_{0}$ and $\operatorname{Im} \tilde{Z}_{0}$. Applying unitarity constraints we can define the maximal muon EDM ${ }^{-5}\left(\left|d_{\mu}\right|_{\text {MAX }}\right)$ as

$$
\left|d_{\mu}\right|_{\mathrm{MAX}}=\frac{\left|d_{A}+d_{B}\right|}{4} \cot \beta\left(1+\tan ^{2} \beta\right)^{1 / 2}+\frac{\left|d_{A}-d_{B}\right|}{4} \tan \beta\left(1+\cot ^{2} \beta\right)^{1 / 2} .
$$

In Figure 3, we present the maximal value allowed by unitarity for the muon and the electron EDMs, as a function of $\tan \beta$ with $m_{0}=100,200$ and $400 \mathrm{GeV}$. Also shown is the experimental upper limit for the electron EDM $\left(\left|d_{e}\right|_{\text {U.L. }}\right)$. 

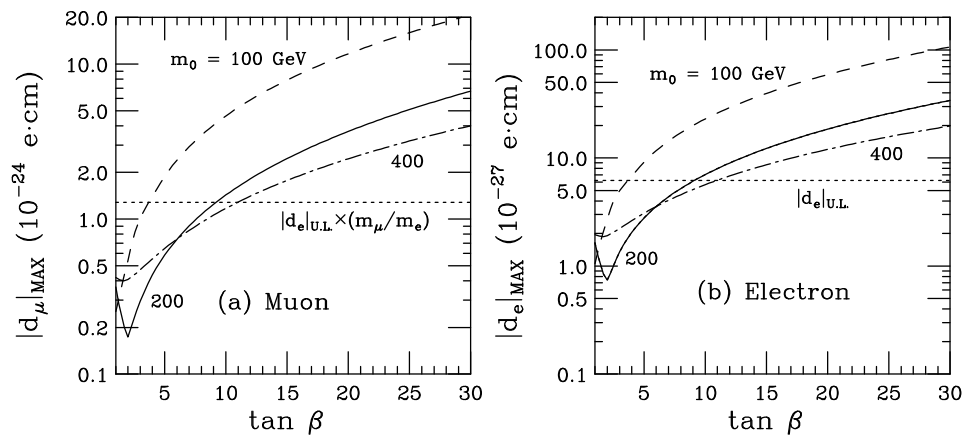

Figure 3: The maximal EDM allowed by unitarity for (a) the muon and (b) the electron.

In this model, for $\tan \beta \gtrsim 10,\left(m_{\mu} / m_{e}\right)\left|d_{e}\right|_{\text {U.L. }}$ could be treated as an upper limit for the muon EDM since $d_{\mu} \approx\left(m_{\mu} / m_{e}\right) d_{e}$. A measured value of the muon EDM above this bound could arise if the muon EDM and the electron EDM are generated from different sources.

\section{Conclusions}

Our results may be summarized as follows 15 :

1. For $\tan \beta \sim 1$, contributions from two loop diagrams involving the $t$ quark and the $W$ boson dominate; while for $\tan \beta \gtrsim 10$, contributions from two loop diagrams involving the $b$ quark and the $\tau$ lepton are dominant.

2. For $\tan \beta \sim 1$ or $\tan \beta \gtrsim 10, d_{\mu} \approx\left(m_{\mu} / m_{e}\right) d_{e}$.

3. For $8 \gtrsim \tan \beta \gtrsim 4$, significant cancellations occur among the contributions from two loop diagrams and the one loop contribution dominates for $\tan \beta \sim 7$.

4. For $\tan \beta>15$ and $m_{0}<300 \mathrm{GeV}$, Higgs-boson exchange could produce a muon EDM which is close to the reach of the proposed BNL experiment.

A positive result for the muon EDM measurement could shed light on nonstandard CP violation. A negative result, however, could mean

1. that $\tan \beta$ is small than 10 ; or,

2. the $\mathrm{CP}$ violation parameters $\operatorname{Im} Z_{0}$, and $\operatorname{Im} \tilde{Z}_{i}$ are smaller than their unitarity bounds; or,

3. the masses of the neutral Higgs scalars and the Higgs pseudoscalar are very close to one another. 9 


\section{Acknowledgments}

This research was supported in part by the U.S. Department of Energy under Grants No. DE-FG05-87ER40319 (Rochester) and No. DE-FG02-95ER40896 (Wisconsin), and in part by the University of Wisconsin Research Committee with funds granted by the Wisconsin Alumni Research Foundation.

\section{References}

1. S.M. Barr and W. Marciano, in CP Violation, ed. C. Jarlskog (World Scientific, Singapore, 1989).

2. W. Bernreuther and M. Suzuki, Rev. Mod. Phys. 63, 313 (1991).

3. F. Hoogeveen, Nucl. Phys. B 341, 322 (1990); I.B. Khriplovich and M. Pospelov, Sov. J. Nucl. Phys. 53, 638 (1991); M. Booth, University of Chicago Report, EFI-93-01, (1993).

4. E. Commins et al., Phys. Rev. A 50, 2960 (1994); K. Abdullah et al., Phys. Rev. Lett. 65, 2347 (1990).

5. J. Bailey et al., J. Phys. G 4, 345 (1978); J. Bailey et al., Nucl. Phys. B 150, 1 (1979).

6. Y. Semertzidis et al., E821 Collaboration at BNL, AGS Expression of Interest: Search for an Electric Dipole Moment of Muon, September 1996.

7. S.L. Glashow and S. Weinberg, Phys. Rev. D 15, 1958 (1977).

8. G.C. Branco and M.N. Rebelo, Phys. Lett. B 160, 117 (1985); J. Liu and L. Wolfenstein, Nucl. Phys. B 289, 1 (1987).

9. S. Weinberg, Phys. Rev. D 42, 860 (1990).

10. S. Barr and A. Zee, Phys. Rev. Lett. 65, 21 (1990).

11. R.G. Leigh, S. Paban and R.-M. Xu, Nucl. Phys. B 352, 45 (1991); J. Gunion and R. Vega, Phys. Lett. B 251, 157 (1990); D. Chang, W.-Y. Keung and T.-C. Yuan, Phys. Rev. D 43, 14 (1991).

12. C. Kao and R.-M. Xu, Phys. Lett. B 296, 435 (1992).

13. CLEO Collaboration, M.S. Alam et al., Phys. Rev. Lett. 74, 2885 (1995).

14. A. Das and C. Kao, Phys. Lett. B 372, 106 (1996).

15. V. Barger, A. Das and C. Kao, Phys. Rev. D 55, 7099 (1997). 